\title{
Bidirectional associations between sedentary time and sleep duration among 12- to 14-year-old adolescents
}

\author{
Maïté Verloigne ${ }^{1 *}$, Veerle Van Oeckel ${ }^{1}$, Ruben Brondeel ${ }^{2}$ and Louise Poppe ${ }^{1}$
}

\begin{abstract}
Background: The aim of this study was to investigate bidirectional associations between (prolonged) sitting time and sleep duration in 12- to 14-year-old adolescents using a between-subjects and within-subjects analyses approach.

Methods: Observational data were used from 108 adolescents (53\% girls; mean age 12.9 (SD 0.7) years) from six schools in Flanders, Belgium. The Axivity AX3 triaxial accelerometer, worn on the thigh, was used to assess daily total sitting time and daily time spent in sedentary bouts of $\geq 30 \mathrm{~min}$ (as a proxy for prolonged sitting time). The Fitbit Charge 3 was used to assess nightly sleep duration. Both monitors were worn on schooldays only (ranging from 4 to 5 days). Linear mixed models were conducted to analyse the associations, resulting in four models. In each model, the independent variable (sleep duration, sitting time or prolonged sitting time) was included as within- as well as between-subjects factor.
\end{abstract}

Results: Within-subjects analyses showed that when the adolescents sat more and when the adolescents spent more time sitting in bouts of $\geq 30$ min than they usually did on a given day, they slept less during the following night ( $p=0.01$ and $p=0.05$ (borderline significant), respectively). These associations were not significant in the other direction. Between-subjects analyses showed that adolescents who slept more on average, spent less time sitting $(p=0.006)$ and less time sitting in bouts of $\geq 30 \mathrm{~min}(p=0.004)$ compared with adolescents who slept less on average. Conversely, adolescents who spent more time sitting on average and adolescents who spent more time sitting in bouts of $\geq 30$ min on average, slept less ( $p=0.02$ and $p=0.003$, respectively).

Conclusions: Based on the between-subjects analyses, interventions focusing on reducing or regularly breaking up sitting time could improve adolescents' sleep duration on a population level, and vice versa. However, the withinsubjects association was only found in one direction and suggests that to sleep sufficiently during the night, adolescents might limit and regularly break up their sitting time the preceding day.

Trial registration: Data have been used from our trial registered at ClinicalTrials.gov (NCT04327414; registered on March 11, 2020).

Keywords: Sitting, Sedentary bouts, Prolonged sitting, Sleep behaviour, Sleep, Youth, Accelerometer, School

\footnotetext{
*Correspondence: maite.verloigne@ugent.be

'Department of Public Health and Primary Care, Ghent University, Corneel Heymanslaan 10, 9000 Ghent, Belgium

Full list of author information is available at the end of the article
}

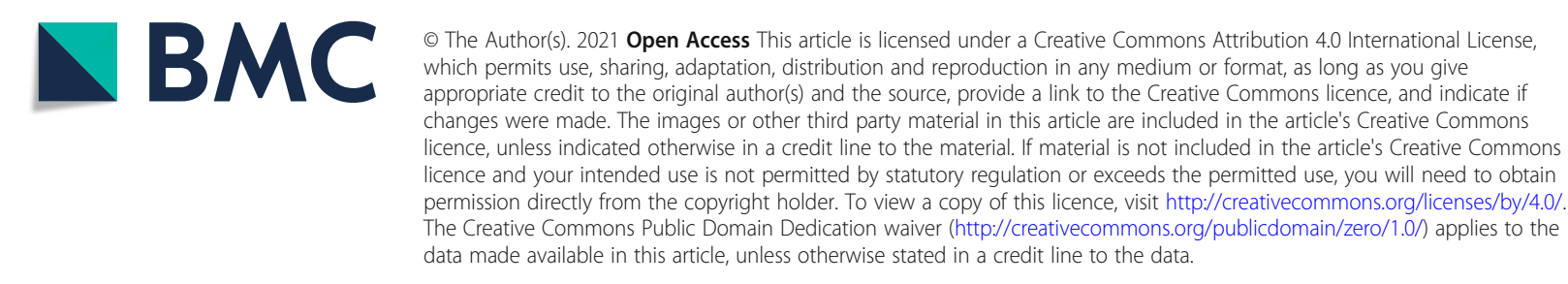




\section{Background}

Adolescents spend more than $60 \%$ of their daily waking hours sedentary [1]. Studies have found that higher levels of sedentary time are associated with more depressive symptoms, a less favourable weight status and decreased physical fitness among adolescents [2, 3]. Nevertheless, the evidence regarding the relation between sedentary time and health outcomes among adolescents is less consistent compared with adults $[4,5]$. Next to the total volume of sedentary time per day, more prolonged sitting may also have a negative impact on adolescents' health. Some studies found that more prolonged sitting is associated with increased cardiometabolic risk factors, a less favourable weight status and decreased physical fitness among youth [6, 7]; however, other studies found limited or no evidence for an association with health above and beyond the association of total sitting time [8,9]. Nevertheless, sedentary behaviour guidelines across the world recommend to regularly break up prolonged sitting in addition to reducing the total sitting time [10].

Sleep behaviour has received increased attention in health promotion research as well. Although there are currently no international sleep guidelines, 14- to 17year-old adolescents are recommended to sleep between 8 and $10 \mathrm{~h}$ per night and younger adolescents even between 9 and $11 \mathrm{~h}$ per night by paediatric sleep experts from the American National Sleep Foundation [11]. However, the proportion of adolescents meeting this recommendation ranges from $32 \%$ to $86 \%$ on schooldays, and $79 \%$ to $92 \%$ on non-schooldays [12]. In addition, these prevalence rates might even be an overestimation, as sleep onset latency (i.e., lying awake in bed before falling asleep) was not considered when calculating the sleep duration [13]. Insufficient sleep is associated with negative health outcomes in adolescents on the short and long term, including depressive symptoms, poor perceived mental health, increased likelihood of substance use [14, 15], and increased risk of developing cardiovascular disease, hypertension, type 2 diabetes and obesity [16].

Previous studies have examined the interrelatedness between both behaviours and suggest a bidirectional, negative association between sedentary time and sleep behaviour $[17,18]$. These studies have applied and reported on a within-subjects analysis approach in order to reveal the acute effects of engaging more or less in a behaviour on a specific day or during a specific night [19]. Currently, there are two studies that have been conducted in adolescents. A study among 15-year-old adolescents found that on days with more sedentary time, adolescents had later sleep onset, shorter sleep duration, but higher sleep maintenance efficiency (i.e. the minutes of actual sleep between sleep onset and offset divided by the sleep duration interval, expressed in percentages) [20]. In the opposite direction, nights with longer sleep duration and later sleep offset, but also nights with a later sleep onset, predicted less sedentary time the day after. Another study in adolescents from the 6th and 8th grade also observed a bidirectional, negative association between sedentary time, screentime and sleep duration, although the association varied by the specific sedentary and sleep health parameter used and by the time period of a day (e.g. sitting time during school hours vs. after school hours) [21]. In both studies sedentary time was measured using hip-worn accelerometers. Although hip-worn accelerometers are reliable and valid tools to measure adolescents' physical (in)activity [22], they are unable to capture body posture [23]. Using physically inactive time as an indicator for sedentary time (i.e. time spent in a sitting position) might lead to mistakenly including standing [23, 24]. To more accurately assess sedentary time, it is highly recommended to use thigh-worn accelerometers [25]. Finally, no accelerometer-based studies among adolescents have examined the bidirectional association between prolonged sedentary time and sleep, although it is important to see whether the relation with sleep duration is different (e.g. stronger).

The aim of this study was to investigate the bidirectional association between objectively measured sedentary time and prolonged sedentary time with sleep duration in 12- to 14-year-old adolescents from Flanders, Belgium, using both a within- and betweensubjects analysis approach. As mentioned before, the within-subjects analysis reveals the immediate effects of engaging more or less in a certain health behaviour, whereas the between-subjects analysis generates general knowledge on behavioural differences between individuals [19]. To unravel the association between the behaviours, it has been recommended to apply both approaches [26]. Our hypothesis was to find bidirectional, negative associations between (prolonged) sedentary time and sleep for the between- and within-subjects analyses.

\section{Methods \\ Study protocol}

For the purpose of this study, baseline data from a standing desks intervention study were analysed. A convenience sample of 22 secondary schools offering general secondary education located in East- and West-Flanders, Belgium, was invited to participate in this study. Twenty-two principals were contacted by email or phone, and six of them agreed to participate. In each school, one class of the 7th or 8th grade with a minimum of 20 pupils was selected by the principal. All children in those classes were invited to participate in the 
study ( $n=132$ ). In total, 123 children (response rate 93\%) with written (parental) consent participated in the baseline measurement (February-March 2020). Measurements involved wearing two devices and completing a questionnaire (including sex and age). Due to COVID19, the intervention and post-test measurement did not take place in Spring 2020. The study protocol was approved by the ethics committee of the Ghent University Hospital (B670201938818). The trial has been registered at ClinicalTrials.gov (NCT04327414; registered on March 11, 2020).

\section{Measurement of sedentary time variables and total volume of physical activity Measurement protocol}

To measure (prolonged) sitting time and total volume of physical activity (as a confounding factor), we used the Axivity AX3 triaxial accelerometer (Axivity, Newcaste, UK), a small waterproof device (dimensions: $23 \times 32.5 \times$ $7.6 \mathrm{~mm} ; 11 \mathrm{~g}$ ). Accelerometers were initialised to measure data at $25 \mathrm{~Hz}$ with $8 \mathrm{G}$ bandwidth. Initialising and downloading was executed via OmGui (version 1.0.0.43, GitHub, Inc., 2020). Adolescents were asked to continuously wear the device for one school week (4 or 5 school days), as the focus of the initial intervention study was on school day behaviour. Adolescents were asked to keep a diary on the time periods during which the device was not worn, if any. These periods of non-wear time were specified by noting the date and time of removal and the date and time of reattachment of the device. Devices were distributed at school on Monday or Tuesday and were collected again on Friday afternoon. The Axivity device was attached to the anterior mid-line of the adolescents' right thigh using 3MTM Tegaderm Transparent Film Roll (3M, St. Paul, MN, USA). The device was oriented with the $\mathrm{x}$-axis vertically (while standing) and the USB port facing down, y-axis horizontally to the left, and the z-axis horizontally forward.

\section{Data processing}

Data were loaded in $\mathrm{R}$ using the open-source software GGIR R package [27]. Non-wear time was detected using GGIR when on at least 1 axis, the standard deviation of the raw accelerometer data was lower than $13 \mathrm{~m}$-gravitational unit $(\mathrm{mg})$ and the range of the data was lower than $150 \mathrm{mg}$ during $60 \mathrm{~min}$ blocks, evaluated per $15 \mathrm{~min}$. In addition, when adolescents reported to have removed the Axivity in their diary, this was manually added as non-wear time during that period of time. Adolescents' data on a daily basis were only included if a minimum of $9 \mathrm{~h}$ of accelerometer data during waking time was available. The waking time used while processing accelerometer data was defined as the time between sleep offset and sleep onset, measured by the Fitbit Charge 3 (see further in the Methods). In case an adolescent had partial missing Fitbit data, missing sleep offset and/or sleep onset was calculated based on the mean sleep offset and/or sleep onset of the other days. When no data regarding sleep offset or sleep onset were available, a fixed sleep offset (i.e. 7 AM) and onset (i.e. 10:30 PM) were used. This method of filling missing data was only used to determine waking time when processing the Axivity data, not to perform the statistical analyses in which sleep duration was used as a variable. In these analyses, missing Fitbit data were retained as missing data. Six types of activity were detected in $5 \mathrm{~s}$ bouts: (a) sitting, (b) lying, (c) standing, (d) walking, (e) cycling, and (f) running (expressed in $\mathrm{min} /$ day) [28]. Then, this variable was recoded as sedentary time (sitting and lying) and non-sedentary time. Total sedentary time per day was calculated by adding the $5 \mathrm{~s}$ bouts of sedentary time. The time spent in sedentary bouts of $\geq 30$ min per day was used as a proxy of prolonged sitting; and calculated by adding the time spent in sedentary bouts of $\geq 30 \mathrm{~min}$ per day. A sedentary bout of $\geq 30 \mathrm{~min}$ was defined as a continuous period of sitting and/or lying time of at least 30 min, with no tolerance (i.e. interruption in the sitting and/or lying activity) allowed. To account for possible differences in wear time between adolescents, the two outcomes were expressed in percentage per day. To ensure the quality of the data, percentages exceeding 90 for sitting time and 81 (i.e. 90\% of 90) for time spent in sedentary bouts of $\geq 30 \mathrm{~min}$ were removed from the analysis. The total volume of physical activity per day was measured as the mean Euclidean Norm Minus One (ENMO). The ENMO metric summarises the triaxial accelerometer data by taking the vector magnitude of the acceleration on the three axes at each observation (i.e. each $0.04 \mathrm{~s}$ ); then extracting $1 \mathrm{~g}$ to accommodate for gravitation, rounding potential negative values to 0 , and multiplying it by 1000 [29].

\section{Measurement of sleep duration Measurement protocol}

The Fitbit Charge 3 (Fitbit, Inc., San Francisco, CA, USA), a wrist-worn activity tracker including a triaxial accelerometer and heart rate monitor, was used to assess adolescents' total sleep duration. Similar as to the Axivity, the Fitbit was continuously worn from Monday or Tuesday until Friday (also during the night). The psychometric properties of the Fitbit Charge 3 are not evaluated yet. However, two studies measured the performance of the Fitbit Charge HR in children and adolescents [30, 31]. The Fitbit Charge HR is the first Fitbit with multi-sensor capability, which is still used in its updated versions such as Charge 3. Both studies showed that the Fitbit Charge HR had an adequate sensitivity (respectively 97\% and 95.7\%) in detecting sleep against 
polysomnography. Nevertheless, the Fitbit Charge HR is not able to detect sleep stages, whereas more newgeneration models (such as the Fitbit Charge 3) combine body movement and heart rate variability to identify and estimate time spent in sleep stages. Although only a very few studies have already evaluated the performance of the new-generation models (and not one of them evaluated the Fitbit Charge 3), it has been concluded that sleep-staging models perform better in assessing sleep behaviour [32].

\section{Data processing}

The Fitbit was synchronised using the Fitbit app (Fitbit, Inc., San Francisco, CA, USA), from which sleep onset and offset were extracted. Sleep onsets between 8 PM and $2 \mathrm{AM}$ and sleep offsets between $5 \mathrm{AM}$ and $9 \mathrm{AM}$ were considered valid, which resulted in excluding 7 days from the analysis. Sleep duration was calculated as the time between sleep onset and offset.

\section{Statistical analyses}

Considering the clustering of the data (i.e., days within participants), linear mixed models with two levels were used for analysing the data. The intraclass correlation (ICC) between observations from the same adolescents was calculated by running intercept-only models with each of the behavioural variables (i.e. sleep duration, percentage of time spent sitting and percentage of time spent sitting in bouts of $\geq 30 \mathrm{~min}$ ) as outcome. The ICC provides information on the proportion of the total variance that is accounted for by grouping the data by participants. Consequently, the ICC reflects the correlation between observations belonging to the same participants.

To estimate the association between adolescents' sleep duration and sedentary time during the following day, a model was fit with sedentary time as dependent variable and sleep duration on the previous night as betweensubject (i.e. mean of the variable at the subject-level) as well as within-subject (i.e. subjects' daily score minus their mean score) independent variables. The distinction between within- and between-subject variables was explicitly made because the standard mixed model approach does not make a distinction between both effects and implicitly assumes these effects are the same. However, incorrectly assuming similar effects can obscure the actual associations (see also Neuhaus and Kalbfleisch [33]). Similarly, for examining the association between sleep duration and prolonged sedentary time during the following day, a model was fit with prolonged sedentary time as dependent variable and sleep duration as between- as well as within-subject independent variables. In both models, adolescents' age, sex and school were also included. To facilitate convergence, the variable 'age' was standardised before it was added to the model. To examine the association between adolescents' sedentary time and sleep duration at the following night, a multilevel model was fit with sleep duration as dependent variable and sedentary time as between- as well as within-subject independent variables. Similarly, to investigate the association between prolonged sedentary time and sleep duration at the following night, a multilevel model was fit with sleep duration as dependent variable and prolonged sedentary time as between- and within-subject independent variables. In both models, adolescents' age, sex, volume of total physical activity and school were also included. The variables 'age' and 'total volume of physical activity' were standardised when entered to the models. Finally, sensitivity analyses were performed to check the impact of deviations from participants' average sleep duration at the night before. To do so, adolescents' average sleep duration was subtracted from their sleep duration at the night before and entered into the model. For each of the fitted models, the assumptions were checked by visually inspecting the residuals versus fitted values plot and the quantile-quantile plot. $P$-values below 0.05 were considered statistically significant and below 0.1 borderline significant. The data were analysed using $R$ version 4.0.3 ( $R$ Development Core Team, 2010), and the R-package lme4 version 1.1-25 [34]. A figure that visually represents the associations analysed in each model has been added as Additional File 1.

\section{Results}

\section{Data availability}

In total, 123 adolescents participated in the study. Two participants lost their Axivity accelerometer, nine participants did not wear a Fitbit during the night, one participant did not provide valid sleep data, two participants did not provide valid sedentary time data and one participant withdrew his/her consent. Hence, the analyses were performed on the data of 108 adolescents. The results of the models focusing on the association between (prolonged) sedentary time and sleep duration at the following night are based on 290 observations from 108 participants. The results of the models focusing on the association between sleep duration and (prolonged) sedentary time the following day are based on 182 observations from 105 participants.

\section{Descriptive statistics}

Sample charachteristics are provided in Table 1. The median number of valid days (i.e. days with $\geq 9 \mathrm{~h}$ of wear time during waking time) of Axivity data was 4 out of a maximum of 5 . The median number of nights during which participants correctly wore the Fitbit was 3 out of a maximum of 4 . The ICC of 0.50 found for sleep time 
Table 1 Characteristics of the sample

\begin{tabular}{llll}
\hline Characteristics & N (\%) / Mean & SD & Range \\
\hline Sex & & & \\
$\quad$ Girls & $57(53)$ & & \\
$\quad$ Boys & $51(47)$ & 0.67 & $12.00-15.00$ \\
Age in years & 12.91 & 0.67 & $4.76-9.88$ \\
Sleep time in hours & 8.37 & 5.50 & $48.53-89.98$ \\
Percentage of waking time spent sitting & 71.34 & 9.12 & $8.80-75.32$ \\
Percentage of waking time spent sitting in bouts of $\geq 30$ min & 36.54 & 0.28 \\
\hline
\end{tabular}

SD Standard deviation, ICC Intraclass correlation between observations within the same adolescents

indicates that the differences between participants in sleep time were similar to the differences detected within participants. The ICCs for the percentage of waking time spent sitting and the percentage of waking time spent in bouts of $\geq 30 \mathrm{~min}$ are very similar, 0.28 and 0.27 respectively. These low ICCs indicate that the variation was mainly accounted for by differences within individuals. Hence, within participants, there was substantial variation in the time spent sedentary (in bouts of $\geq 30 \mathrm{~min}$ ) from day to day.

\section{The association between sedentary time and sleep duration at the following night}

Table 2 presents the results of the model examining the association between the percentage of waking time spent sitting and sleep duration at the following night. This model did not adequately converge when the variable "school" was included. However, because similar results were obtained by removing school from the model, we decided to report the results of the original model. The significant within-subject association between sedentary time and sleep duration at the following night (beta(SE) $=-0.02(0.01) ; p=$ 0.01 ) indicates that, when the adolescents sat more than they usually did on a given day, they slept less during the following night. This association was independent of their total volume of physical activity that day and their average sedentary time. Furthermore, a significant between-subject association between sedentary time and sleep duration the following night was detected $(\operatorname{beta}(\mathrm{SE})=-0.03(0.01) ; p=0.02)$, indicating that adolescents who spent more time sitting on average slept less compared with adolescents who spent less time sitting on average. Similar results were obtained with the sensitivity analysis including adolescents' deviation in sleep duration at the night before as predictor (see Additional file 2).

\section{The association between prolonged sedentary time and sleep duration at the following night}

A borderline significant within-subject association between prolonged sedentary time and sleep duration at the following night was identified (beta $(\mathrm{SE})=-$ $0.01(0.004) ; p=0.05$; see Table 2$)$. This indicates that, when adolescents spent more time sitting in bouts of $\geq 30 \mathrm{~min}$, they slept less during the following night. This borderline significant association was independent from their level of physical activity and their average sitting time. Furthermore, adolescents who spent on average more time sitting in bouts of $\geq 30$ min slept less than adolescents who spent on average less time sitting in bouts of $\geq 30 \mathrm{~min}(\mathrm{beta}(\mathrm{SE})=-0.02(0.01) ; p=0.003)$. Similar results were obtained with the sensitivity analysis including adolescents' deviation in sleep duration at the night before as predictor (see Additional file 2).

Table 2 Results of the model examining the association between (prolonged) sedentary time and sleep duration at the following night

\begin{tabular}{lll}
\hline & Sleep duration & \\
\cline { 2 - 3 } Independent variables & Regression coefficient & 95\% confidence interval \\
\hline (Intercept) & 10.48 & $8.76 ; 12.20^{* * *}$ \\
Sedentary time (within-subject) & -0.02 & $-0.04 ;-0.005^{*}$ \\
Sedentary time (between-subject) & -0.03 & $-0.06 ;-0.005^{*}$ \\
(Intercept) & 9.10 & $8.54 ; 9.65^{* * *}$ \\
Prolonged sedentary time (within-subject) & -0.01 & $-0.02 ;-0.0002^{*}$ \\
Prolonged sedentary time (between-subject) & -0.02 & $-0.04 ;-0.008^{* *}$ \\
\hline
\end{tabular}

${ }^{* * *} p<.001{ }^{* *} p<.01{ }^{*} p<.05^{*} p<.10$; Controlled for age, sex, total volume of physical activity, and school 
The association between sleep duration and sedentary time the following day

A significant association between adolescents' mean sleep duration and sitting time was detected (beta $(\mathrm{SE})=$ $-2.30(0.83) ; p=0.006$; see Table 3$)$, indicating that adolescents who slept more on average spent less time sitting than adolescents who slept less on average.

\section{The association between sleep duration and prolonged sedentary time the following day}

A significant between-subject association between sleep duration and prolonged sedentary time was detected $(\operatorname{beta}(\mathrm{SE})=-4.60(1.58) ; p=0.004$; see Table 3$)$, indicating that adolescents who slept more on average spent less time sitting in bouts of $\geq 30 \mathrm{~min}$ than adolescents who slept less on average.

\section{Discussion}

The aim of this study was to investigate bidirectional associations between (prolonged) sedentary time and sleep duration in 12- to 14-year old adolescents from Flanders, Belgium. Within-subject results showed that adolescents who sit more than they normally do on a specific school day, sleep less the following night. In addition, adolescents who engage in more prolonged sitting than they normally do on a specific school day, also sleep less the following night, although the association was only borderline significant.

Although various mechanisms to explain the acute effect of physical activity on sleep have already been suggested [35], there is not much evidence on the mechanisms explaining the acute effect of sitting on sleep. Therefore, we suggest some hypotheses below, but future research should verify them. A first possible explanation could be that specific factors mediate the association between more (prolonged) sitting and less sleep. For example, high levels of sedentary time and engaging in long sedentary bouts are related to negative emotions in adolescents, such as feeling unwell or stress [36]. Experiencing negative emotions has in turn an impact on adolescents' sleep onset, resulting in a short sleep duration [37]. Another example is that more sitting and more prolonged sitting (i.e. less breaks in sitting time) on a given day are both associated with less energy expenditure [38]. This could result in feeling less sleepy in the evening and not feeling the urge to go to bed [20]. This might especially be detrimental for adolescents, as biologically they already have an evening circadian phase preference for later bed times [39]. So in terms of sleeping sufficiently during the night, an important and useful strategy for adolescents might thus be to not only limit but also to break up the time spent sedentary the preceding day. Another explanation for the acute effect could be that the additional sedentary time of the adolescents on a specific day is spent in the form of screen-time activities during the evening period [40]. This would imply that it is not the sitting behaviour itself that is important for sleeping sufficiently the following night, but rather the activity that is done while sitting. Indeed, screen-time activities are an important source of entertainment and might therefore push adolescents to postpone their bed time [41]. This results in a shorter sleep duration, as rise time is mostly fixed on schooldays. Additionally, such screen-time activities can involve long bouts of sedentary time, such as binge watching, which explains the specific association between prolonged sitting and sleep duration. Extra analyses conducted as part of this study confirm that it is the additional (prolonged) sedentary time during non-school hours that is significantly related to a shorter sleep duration, and not the additional (prolonged) sedentary time during school hours (see Additional file 3). This is in line with the study of Kim and colleagues [21] in which more sedentary time during the after-school period, and not during the school period, was associated with a shorter sleep duration the following night among young adolescents. This means that when promoting the reduction and breaking up of sedentary time during the day, there should be a focus on the nonschool hours.

Unexpectedly, the association was not found in the other direction: sleep duration during the night was not significantly related to more sedentary time or to more time in long sedentary bouts on the following day. However, other studies found the association in both directions (e.g. $[17,20]$ ), suggesting our results

Table 3 Results of the model examining the association between sleep duration and (prolonged) sedentary time the following day

\begin{tabular}{llllll}
\hline & Sedentary time & & & Prolonged sedentary time \\
\cline { 2 - 3 } \cline { 5 - 6 } Independent variables & Regression coefficient & $\mathbf{9 5 \%}$ confidence interval & & Regression coefficient & 95\% confidence interval \\
\hline (Intercept) & 86.00 & $72.32 ; 99.67^{* * *}$ & 69.08 & $43.35 ; 95.34^{* * *}$ \\
Sleep duration (within-subject) & 0.57 & $-1.60 ; 2.74$ & -0.09 & $-3.83 ; 3.74$ \\
Sleep duration (between-subject) & -2.30 & $-3.88 ;-0.72^{* *}$ & -4.60 & $-7.64 ;-1.63^{* *}$ \\
\hline
\end{tabular}

***p $<.001 * * p<.01$; Controlled for age, sex, and school 
do not completely align. A possible reason why sleep on a given night did not affect sedentary time the next day in our study, might be that we only collected data on school days. Previous research has attributed some of the within-variability among adolescents to the different sleeping patterns between weekdays and weekend days (i.e. catching up the loss of sleep from the school week during the weekend) [42], suggesting that there might be less withinvariability between the different weekdays. In addition, our study sample included relatively young adolescents, and adolescents' age has shown to be a positive predictor of the within-variability in total sleep duration [42, 43]. However, one of the studies that found an association between sleep duration on a given night and sedentary time the following day also only included school days in young adolescents, suggesting other factors might also be relevant to explain the difference between study findings. The difference in measurement method could play a role here, as previous studies have used hip-worn accelerometers to measure both sedentary time as well as sleep [17, 20]. However, hip-worn accelerometers cannot accurately distinguish standing from sitting [23, 24], suggesting our estimates of time spent sitting are more accurate than the estimates of other studies. For sleep, it is less clear if hip- or wrist-worn devices are preferred, although sleep has been validated mainly with wrist-worn devices [44]. Hip-worn devices have been shown to overestimate sleep duration compared to wrist-worn devices [45], however, others have stated that hip-worn devices are superior to assess sleep duration [46].

In contrast to the within-subjects results, the between-subjects analyses revealed a bidirectional association. Adolescents who, on average, sleep less, have higher levels of sedentary time and spend more time in prolonged sitting. It was also found that adolescents who, on average, have higher levels of sedentary time or spend more time in prolonged sitting, have a shorter sleep duration. These two modifiable health behaviours are thus in general interrelated, which means future interventions focusing on reducing or regularly breaking up sedentary time could improve adolescents' sleeping behaviour on a population level, and vice versa. This can be important for age-specific obesity prevention programmes, as sedentary time and sleep have been identified as two factors influencing its development [2, 16]. This would imply that the health effect of population-based interventions focusing on one behaviour (sedentary time or sleep) might be reinforced because of a concurrent effect on the other behaviour. However, it must be acknowledged that effect sizes were relatively small.
A first strength of this study is that both withinsubjects as between-subjects analyses were conducted in order to unravel the association between sleep and sedentary time in adolescents. Between-subjects analyses inform us about inter-individual differences or in other words, the general interrelatedness of sedentary time and sleep duration (i.e. on a trait level), whereas withinsubjects analyses inform us about intra-individual differences or the immediate effects of sitting/sleeping more or less than usual (i.e. on a state level) [19]. Another strength is the inclusion of spending time in sedentary bouts of at least $30 \mathrm{~min}$ as a proxy for prolonged sitting on a day. Previous studies have only investigated relationships between levels of sedentary time and sleep [17, $20,21]$, and it has been advocated to examine the associations between breaks in sedentary time and sleep as well [26]. The current study adds to the literature by showing that not only the total time spent sedentary, but also how it is accumulated is important for adolescents' sleep. A final strength is the use of device-based instruments to objectively measure sitting time, the time spent sitting in bouts of at least $30 \mathrm{~min}$ and sleep duration. Moreover, we used a thigh-worn accelerometer, which is more accurate to estimate people's sedentary time compared to a hip-worn accelerometer [25]. However, the disadvantage is that there is no information on the specific sedentary activities performed, suggesting future research should combine different measurement methods. Study limitations include the convenience sampling method and specific focus on secondary schools offering general education, limiting the representativeness of our findings. Another limitation is that we only had data on school days because of the nature of the larger schoolbased intervention study from which data were used. A final limitation is that we did not have day-to-day data on adolescents' sleep quality. Although sleep duration and sleep quality are inherently associated with each other, it has been advocated that sleep quality is the most important health parameter [47, 48]. Future studies could thus use ecological momentary assessment methods to assess adolescents' sleep quality.

\section{Conclusions}

The between-subjects analyses in this study showed a bidirectional, negative association between (prolonged) sedentary time and sleep duration on weekdays in 12- to 14-year-old adolescents, suggesting population-based interventions focusing on a general improvement of one behaviour might affect the other health behaviour. However, the within-subjects analyses only revealed a negative association between (prolonged) sedentary time and sleep duration, but not in the other direction. This suggests that more sitting and more prolonged sitting than 
normally on a weekday has an immediate, negative effect on the sleep duration the following night. This might be an important public health message for an adolescent population. Future research could disentangle the mechanisms underlying these associations.

\section{Abbreviations}

ENMO: Euclidean Norm Minus One; SD: Standard deviation; SE: Standard error; ICC: Intraclass correlation between observations within the same adolescents

\section{Supplementary Information}

The online version contains supplementary material available at https:/doi. org/10.1186/s12889-021-11694-9.

Additional file 1: A visual representation of the four statistical models.

Additional file 2: Results of the sensitivity analysis examining the association between (prolonged) sedentary time and sleep duration at the following night.

Additional file 3: Results of the models examining the association between (prolonged) sedentary time at school/elsewhere and sleep duration at the following night.

\section{Acknowledgements}

Not applicable.

\section{Authors' contributions}

MV and LP developed the concept of this manuscript, WO conducted the data collection, RB was involved in data processing, LP conducted the statistical analyses (supported by RB). All authors were significantly involved in writing specific parts of the manuscript, critically revising the manuscript for important intellectual content and have read and approved the final manuscript.

\section{Funding}

This study was granted by a Research Foundation Flanders (FWO) Research Grant (1505519 N). RB is supported by the Research Foundation Flanders (FWO; postdoctoral research fellowship).

\section{Availability of data and materials}

The datasets and R-script used and/or analysed during the current study are available from the corresponding author on reasonable request.

\section{Declarations}

\section{Ethics approval and consent to participate}

All protocols are carried out in accordance with relevant guidelines and regulations. We obtained written informed consent from all children that participated in this study and one of their parents. Participants were made fully aware of the study. The study protocol was approved by the ethics committee of the Ghent University Hospital (B670201938818)

\section{Consent for publication}

Not applicable.

\section{Competing interests}

The authors declare that they have no competing interests.

\section{Author details}

${ }^{1}$ Department of Public Health and Primary Care, Ghent University, Corneel Heymanslaan 10,9000 Ghent, Belgium. ${ }^{2}$ Department of Movement and Sports Sciences, Ghent University, Watersportlaan 10, 9000 Ghent, Belgium.
Received: 6 May 2021 Accepted: 29 August 2021

Published online: 15 September 2021

\section{References}

1. Arundell L, Salmon J, Koorts H, Contardo Ayala AM, Timperio A. Exploring when and how adolescents sit: cross-sectional analysis of activPALmeasured patterns of daily sitting time, bouts and breaks. BMC Public Health. 2019;19(1):653. https://doi.org/10.1186/s12889-019-6960-5.

2. van Ekris E, Altenburg TM, Singh AS, Proper Kl, Heymans MW, Chinapaw MJ. An evidence-update on the prospective relationship between childhood sedentary behaviour and biomedical health indicators: a systematic review and meta-analysis. Obes Rev. 2016;17(9):833-49. https://doi.org/10.1111/ obr.12426.

3. Kandola A, Lewis G, Osborn DPJ, Stubbs B, Hayes JF. Depressive symptoms and objectively measured physical activity and sedentary behaviour throughout adolescence: a prospective cohort study. Lancet Psychiatry. 2020;7(3):262-71. https://doi.org/10.1016/\$2215-0366(20)30034-1.

4. Chinapaw M, Altenburg T, Brug J. Sedentary behaviour and health in children - evaluating the evidence. Prev Med. 2015;70:1-2. https://doi.org/1 0.1016/j.ypmed.2014.10.029.

5. Chinapaw MJ, Proper Kl, Brug J, van Mechelen W, Singh AS. Relationship between young peoples' sedentary behaviour and biomedical health indicators: a systematic review of prospective studies. Obes Rev. 2011;12(7): e621-32. https://doi.org/10.1111/j.1467-789X.2011.00865.x.

6. Bailey DP, Charman SJ, Ploetz T, Savory LA, Kerr CJ. Associations between prolonged sedentary time and breaks in sedentary time with cardiometabolic risk in 10-14-year-old children: the HAPPY study. J Sports Sci. 2017;35(22):2164-71. https://doi.org/10.1080/02640414.2016.1260150.

7. Mann KD, Howe LD, Basterfield L, Parkinson KN, Pearce MS, Reilly JK, et al. Longitudinal study of the associations between change in sedentary behavior and change in adiposity during childhood and adolescence: Gateshead millennium study. Int J Obes. 2017;41(7):1042-7. https://doi.org/1 0.1038/ijo.2017.69.

8. Cliff DP, Hesketh KD, Vella SA, Hinkley T, Tsiros MD, Ridgers ND, et al. Objectively measured sedentary behaviour and health and development in children and adolescents: systematic review and meta-analysis. Obes Rev. 2016;17(4):330-44. https://doi.org/10.1111/obr.12371.

9. Verswijveren S, Lamb KE, Bell LA, Timperio A, Salmon J, Ridgers ND. Associations between activity patterns and cardio-metabolic risk factors in children and adolescents: a systematic review. PLoS One. 2018;13(8): e0201947. https://doi.org/10.1371/journal.pone.0201947.

10. Australian Government. Available from: https://www.health.gov.au/healthtopics/physical-activity-and-exercise/physical-activity-and-exerciseguidelines-for-all-australians?utm_source=health.gov.au\&utm_medium=ca llout-auto-custom\&utm_campaign=digital_transformation.

11. Hirshkowitz M, Whiton K, Albert SM, Alessi C, Bruni O, DonCarlos L, et al. National Sleep Foundation's updated sleep duration recommendations: final report. Sleep Health. 2015;1(4):233-43. https://doi.org/10.1016/j.sleh.2015.10. 004.

12. Gariepy G, Danna S, Gobina I, Rasmussen M, Gaspar de Matos M, Tynjälä J, et al. How are adolescents sleeping? Adolescent sleep patterns and sociodemographic differences in 24 European and north American countries. J Adolesc Health. 2020;66(6s):S81-s8. https://doi.org/10.1016/j.ja dohealth.2020.03.013.

13. Vandendriessche A, Dierckens M, Delaruelle K, Deforche B. How are adolescents sleeping? Conservative estimates of sleep duration underestimate the problem. J Adolesc Health. 2021;68(4):830. https://doi. org/10.1016/j.jadohealth.2020.12.137.

14. Gradisar M, Gardner G, Dohnt H. Recent worldwide sleep patterns and problems during adolescence: a review and meta-analysis of age, region, and sleep. Sleep Med. 2011;12(2):110-8. https://doi.org/10.1016/j.sleep.201 0.11 .008 .

15. Shochat T, Cohen-Zion M, Tzischinsky O. Functional consequences of inadequate sleep in adolescents: a systematic review. Sleep Med Rev. 2014; 18(1):75-87. https://doi.org/10.1016/j.smrv.2013.03.005.

16. Aldabal L, Bahammam AS. Metabolic, endocrine, and immune consequences of sleep deprivation. Open Respir Med J. 2011:5(1):31-43. https://doi.org/10.2174/1874306401105010031.

17. Lin Y, Tremblay MS, Katzmarzyk PT, Fogelholm M, Hu G, Lambert EV, et al. Temporal and bi-directional associations between sleep duration and physical activity/sedentary time in children: an international 
comparison. Prev Med. 2018;111:436-41. https://doi.org/10.1016/j. ypmed.2017.12.006

18. Nixon GM, Thompson JM, Han DY, Becroft DM, Clark PM, Robinson E, et al. Short sleep duration in middle childhood: risk factors and consequences. Sleep. 2008;31(1):71-8. https://doi.org/10.1093/sleep/31.1.71.

19. Kanning MK, Ebner-Priemer UW, Schlicht WM. How to investigate withinsubject associations between physical activity and momentary affective states in everyday life: a position statement based on a literature overview. Front Psychol. 2013;4:187. https://doi.org/10.3389/fpsyg.2013.00187.

20. Master L, Nye RT, Lee S, Nahmod NG, Mariani S, Hale L, et al. Bidirectional, daily temporal associations between sleep and physical activity in adolescents. Sci Rep. 2019;9(1):7732. https://doi.org/10.1038/s41598-019-44 059-9.

21. Kim Y, Umeda M, Lochbaum M, Sloan RA. Examining the day-to-day bidirectional associations between physical activity, sedentary behavior, screen time, and sleep health during school days in adolescents. PLoS One. 2020;15(9):e0238721. https://doi.org/10.1371/journal.pone.0238721.

22. Cleland I, Kikhia B, Nugent C, Boytsov A, Hallberg J, Synnes K, et al. Optimal placement of accelerometers for the detection of everyday activities. Sensors (Basel). 2013;13(7):9183-200. https://doi.org/10.3390/s130709183.

23. Berendsen BA, Hendriks MR, Meijer K, Plasqui G, Schaper NC, Savelberg HH. Which activity monitor to use? Validity, reproducibility and user friendliness of three activity monitors. BMC Public Health. 2014;14(1):749. https://doi. org/10.1186/1471-2458-14-749.

24. Janssen X, Cliff DP. Issues related to measuring and interpreting objectively measured sedentary behavior data. Meas Phys Educ Exerc Sci. 2015;19(3): 116-24. https://doi.org/10.1080/1091367X.2015.1045908.

25. Brønd JC, Grøntved A, Andersen LB, Arvidsson D, Olesen LG. Simple Method for the Objective Activity Type Assessment with Preschoolers, Children and Adolescents. Children (Basel). 2020;7(7):72.

26. Choi H, Kim C, Ko H, Park CG. Relationship between sedentary time and sleep duration among Korean adolescents. J Sch Nurs. 2019; 1059840519842230(6):423-9. https://doi.org/10.1177/1059840519842230.

27. Van Hees VThzorY. Available from: https://zenodo.org/record/4640382 \#.YGbXpugzaUk.

28. Narayanan A, Stewart T, Mackay L. A dual-accelerometer system for detecting human movement in a free-living environment. Med Sci Sports Exerc. 2020:52(1):252-8. https://doi.org/10.1249/MSS.0000000000002107.

29. van Hees VT, Gorzelniak L, Dean León EC, Eder M, Pias M, Taherian S, et al. Separating movement and gravity components in an acceleration signal and implications for the assessment of human daily physical activity. PLoS One. 2013;8(4):e61691. https://doi.org/10.1371/journal.pone.0061691.

30. de Zambotti M, Baker FC, Willoughby AR, Godino JG, Wing D, Patrick K, et al. Measures of sleep and cardiac functioning during sleep using a multisensory commercially-available wristband in adolescents. Physiol Behav. 2016;158:143-9. https://doi.org/10.1016/j.physbeh.2016.03.006

31. Godino JG, Wing D, de Zambotti M, Baker FC, Bagot K, Inkelis S, et al. Performance of a commercial multi-sensor wearable (Fitbit charge HR) in measuring physical activity and sleep in healthy children. PLoS One. 2020; 15(9):e0237719. https://doi.org/10.1371/journal.pone.0237719.

32. Haghayegh S, Khoshnevis S, Smolensky MH, Diller KR, Castriotta RJ. Accuracy of wristband Fitbit models in assessing sleep: systematic review and Metaanalysis. J Med Internet Res. 2019;21(11):e16273. https://doi.org/10.2196/16273.

33. Neuhaus JM, Kalbfleisch JD. Between- and within-cluster covariate effects in the analysis of clustered data. Biometrics. 1998;54(2):638-45. https://doi. org/10.2307/3109770.

34. Bates D, Mächler M, Bolker B, Walker S. Fitting Linear Mixed-Effects Models Using Ime4. ArXiv e-prints. 2014;arXiv:1406.

35. Kredlow MA, Capozzoli MC, Hearon BA, Calkins AW, Otto MW. The effects of physical activity on sleep: a meta-analytic review. J Behav Med. 2015;38(3): 427-49. https://doi.org/10.1007/s10865-015-9617-6.

36. Giurgiu M, Koch ED, Ottenbacher J, Plotnikoff RC, Ebner-Priemer UW, Reichert M. Sedentary behavior in everyday life relates negatively to mood: an ambulatory assessment study. Scand J Med Sci Sports. 2019;29(9):134051. https://doi.org/10.1111/sms.13448.

37. Gruber R, Somerville G, Paquin S, Boursier J. Determinants of sleep behavior in adolescents: a pilot study. Sleep Health. 2017;3(3):157-62. https://doi. org/10.1016/j.sleh.2017.03.004.

38. Swartz AM, Squires L, Strath SJ. Energy expenditure of interruptions to sedentary behavior. Int J Behav Nutr Phys Act. 2011:8(1):69. https://doi.org/1 $0.1186 / 1479-5868-8-69$
39. Owens J. Insufficient sleep in adolescents and young adults: an update on causes and consequences. Pediatrics. 2014;134(3):e921-32. https://doi.org/1 0.1542/peds.2014-1696.

40. Haycraft E, Sherar LB, Griffiths P, Biddle SJH, Pearson N. Screen-time during the after-school period: a contextual perspective. Prev Med Rep. 2020;19: 101116. https://doi.org/10.1016/.jpmedr.2020.101116.

41. Hale L, Kirschen GW, LeBourgeois MK, Gradisar M, Garrison MM, Montgomery-Downs $\mathrm{H}$, et al. Youth screen media habits and sleep: sleepfriendly screen behavior recommendations for clinicians, educators, and parents. Child Adolesc Psychiatr Clin N Am. 2018;27(2):229-45. https://doi. org/10.1016/j.chc.2017.11.014.

42. Blunden S, Magee C, Clarkson L, Searle A, Banks S, Olds T. Interindividual and intraindividual variability in adolescent sleep patterns across an entire school term: a pilot study. Sleep Health. 2019;5(6):546-54. https://doi.org/1 0.1016/j.sleh.2019.08.002.

43. Moore M, Kirchner HL, Drotar D, Johnson N, Rosen C, Redline S. Correlates of adolescent sleep time and variability in sleep time: the role of individual and health related characteristics. Sleep Med. 2011;12(3):239-45. https://doi. org/10.1016/j.sleep.2010.07.020.

44. Meltzer LI, Montgomery-Downs HE, Insana SP, Walsh CM. Use of actigraphy for assessment in pediatric sleep research. Sleep Med Rev. 2012;16(5):46375. https://doi.org/10.1016/j.smrv.2011.10.002.

45. Hjorth M, Chaput J-P, Damsgaard C, Dalskov S-M, Michaelsen K, Tetens l, et al. Measure of sleep and physical activity by a single accelerometer: can a waist-worn Actigraph adequately measure sleep in children? Sleep Biol Rhythms. 2012;10(4):328-35. https://doi.org/10.1111/ j.1479-8425.2012.00578.x.

46. Smith C, Galland B, Taylor R, Meredith-Jones K. ActiGraph GT3X+ and Actical wrist and hip worn accelerometers for sleep and wake indices in young children using an automated algorithm: validation with polysomnography. Front Psychiatry. 2019;10:958. https://doi.org/10.3389/fpsyt.2019.00958.

47. Bin YS. Is sleep quality more important than sleep duration for public health? Sleep. 2016;39(9):1629-30. https://doi.org/10.5665/sleep.6078.

48. Cappuccio FP, D'Elia L, Strazzullo P, Miller MA. Quantity and quality of sleep and incidence of type 2 diabetes: a systematic review and meta-analysis. Diabetes Care. 2010;33(2):414-20. https://doi.org/10.2337/dc09-1124.

\section{Publisher's Note}

Springer Nature remains neutral with regard to jurisdictional claims in published maps and institutional affiliations.
Ready to submit your research? Choose BMC and benefit from:

- fast, convenient online submission

- thorough peer review by experienced researchers in your field

- rapid publication on acceptance

- support for research data, including large and complex data types

- gold Open Access which fosters wider collaboration and increased citations

- maximum visibility for your research: over $100 \mathrm{M}$ website views per year

At $\mathrm{BMC}$, research is always in progress.

Learn more biomedcentral.com/submissions 